\title{
A THREE-MONTH LONGITUDINAL STUDY FOR DIAGNOSTIC STABILITY OF ACUTE AND TRANSIENT PSYCHOTIC DISORDER
}

\author{
Devavrat Joshi' ${ }^{*}$, Ananta Prasad Adhikari', Praveen Bhattarai², Pawan Sharma ${ }^{3}$ \\ ${ }^{1}$ Department of Psychiatry, National Academy of Medical Sciences (NAMS), Mental Hospital, Lagankhel, Lalitpur. \\ ${ }^{2}$ Department of Psychiatry, Mental Hospital, Lagankhel, Lalitpur. ${ }^{3}$ Deaprtment of Psychiatry, Patan Academy of Health Sciences (PAHS), Lagankhel, Lalitpur
}

spondence to: Dr. Devavrat Joshi, Department of Psychiatry, National Academy of Medical Sciences (NAMS), Mental Hospital, Lagankhel, Lalitpur.

Email:devratjoshi@yahoo.com

\begin{abstract}
Background: The diagnostic category of "acute and transient psychotic disorders" (ATPD) comprises of psychotic disorders with features of acute onset (within 2 weeks), presence of typical syndromes that are either polymorphic or schizophrenic or persistently delusional, evidence for associated acute stress and complete recovery in most cases within 2-3 months. Studies of diagnostic stability among patients with the initial diagnosis of ATPD at their first admission have reported modest stabilities spanning over different periods of time, with higher levels of stability reported in samples from the developing countries. This study aimed at investigating the diagnostic stability of cases of ATPD at the Mental Hospital, Lagankhel over a period of 3 months from the first diagnosis. Methods: ASeventy-five drug naïve patients with the initial diagnosis of first episode ATPD admitted at the in-patient unit of Mental Hospital, Lagankhel were followed up longitudinally and their diagnosis was assessed independently by two consultant psychiatrists over a period of 3 months. Results: Average age of the sample was 30.71 years. $66.7 \%$ of the cases retained their index diagnosis of ATPD at 3 months, while the remaining required diagnostic revision. A higher number of patients had their diagnosis revised to Affective Disorders including Mania and Severe depression with psychotic features (13.88\%) when compared to Schizophrenia (6.94\%). Conclusion: ATPD is a relatively stable diagnostic category in the Nepalese Scenario as well with more than two third of the patients retaining the initial diagnosis at 3 months.
\end{abstract}

Key words: Diagnostic Stability, Psychosis, ATPD, Diagnostic revision.

\section{INTRODUCTION}

The diagnostic category of "acute and transient psychotic disorders" (ATPD) as introduced by ICD-10(F23, WHO 1992) comprises psychotic disorders with features of acute onset (within 2 weeks), presence of typical syndromes that are either polymorphic or schizophrenic or persistently delusional, evidence for associated acute stress and complete recovery in most cases within $2-3$ months. ${ }^{1}$ In creating this category, the authors of ICD-10 took account of a number of concepts for such psychotic disorders,including cycloid psychosis in German psychiatry, bouffée délirante in French psychiatry, psychogenic or reactive psychosis in Scandinavian psychiatry, the remitting schizophrenia in American psychiatry,the atypical psychosis in Japanese psychiatry or acute psychosis of uncertain origin in
Indian psychiatry. ${ }^{2}$ The confirmatory evidence for the validity of this diagnostic entity has come from the international initiatives in the form of WHO multicentered collaborative studies directed primarily at the study of schizophrenia (IPSS) ${ }^{3}$, first onset psychosis (DOSMeD) ${ }^{4}$ and Acute Psychoses (CAP) ${ }^{5}$. Studies of diagnostic stability among patients with the initial diagnosis of ATPD at their first admission have reported modest stabilities spanning over periods of time. ${ }^{6,7}$

Diagnostic stability, a measure of the degree to which a diagnosis remains the same at the subsequent assessments of the patient implies that the followup diagnosis is the same as the baseline diagnosis, but it does not necessarily mean that the patient is 
still symptomatic of the disorder at follow-up. There are many factors that lead to diagnostic changes in acute psychosis like poor reliability of assessment, sources of new information (such as informants), longitudinal observation of the symptoms and/or the evolution of the illness. ${ }^{8}$ Similarly the psychotic symptoms can be part of schizophrenia spectrum and affective disorders, as well as induced by substance abuse or structural brain lesions; this further underlines the need to verify diagnostic stability following the initial diagnosis of a psychotic disorder. ${ }^{9}$

Considering the incidence of ATPD in the clinical setting of Nepal and the need of validating the the diagnosis over a period of time, the current study has tried to explore the socio-demographic correlates of this disorder and short term diagnostic stability over a period of 3 months from their first diagnosis, in a sample of patients from the tertiary care center of Nepal.

\section{METHOD}

A total of 75 subsequent drug naïve admissions of either gender in inpatient unit of the Mental Hospital,
Lagankhel Nepal, from June to August 2016, aged $>=15$ years with the initial diagnosis of Acute and Transient Psychotic Disorders (ATPD) were included. The diagnosis of ATPD was made as per the ICD 10 diagnostic criteria at admission and confirmed independently by two consultant Psychiatrists. The patients were enrolled after taking informed consent of their attendants. The socio-demographic profile was collected using a semi structured proforma and the diagnosis was made clinically. Diagnosis of the participants was reviewed at discharge and at follow up visits at 1 and 3 months after the initial diagnosis.

\section{RESULTS}

Among 75 patients one patient was taken away from the hospital against medical advice, on the day of admission itself for seeking treatment from faith healers. Additionally, two patients absconded from the hospital on the $3^{\text {rd }}$ and $9^{\text {th }}$ day of admission respectively and thus could not be followed up. Hence, 72 out of the 75 patients were either formally discharged upon improvement of their condition or were referred to some other center in case of identification of some underlying organic cause.

Table 1: Socio-demographic variables

\begin{tabular}{|l|l|l|}
\hline Variables & Number (75) & Percentage (\%) \\
\hline Sex & & \\
Male & 37 & 49.3 \\
Female & 38 & 50.7 \\
\hline Mean Age & $30.71 \pm 14.62$ & NA \\
\hline Residence & & \\
Kathmandu valley & 13 & 17.3 \\
Outside Kathmandu valley & 62 & 82.7 \\
\hline Education & & \\
Illiterate & 6 & 8 \\
Primary level & 17 & 22.7 \\
Secondary & 44 & 58.7 \\
Graduation or higher & 8 & 10.7 \\
\hline Duration of Hospital Stay & & \\
Less than 15 days & 5 & 68 \\
More than 15 days & 2 & 32 \\
Average Duration of Hospital Stay & $14.98 \pm 13.95$ days & \\
\hline
\end{tabular}


As seen in Table1 the distribution of male and female were almost equal in the sample with age ranging from 16 to 80 years with a mean age of 30.71 years ( $S D=14.62$ years). Majority of the participants (58.7\%) had attained education up to secondary level. Majority (82.7\%) of the sample population lived outside of Kathmandu valley indicating the huge area of coverage for service from the center. The mean duration of hospital stay was $14.98 \pm$ 13.95 days with majority (68\%) leaving hospital in less than 15 days.

At the time of discharge from the hospital, the diagnosis of ATPD remained the same in 54 out of 72 (75\%) patients. Similarly, at one month follow up, the diagnosis of ATPD remained unchanged in 52 out of 72 (72.2\%) patients, whereas at three months follow up 48 out of 72 patients (66.7\%) retained the diagnosis of ATPD. However, at the end of three months 5 patients were either referred to neurologists or were lost to follow-up. As depicted in Table 2, three patients were found to be having a demonstrable underlying intracranial pathology giving rise to the psychotic symptoms, hence, their diagnoses was changed to either 'Delirium' (F 5.0) or Other mental disorders due to brain damage and dysfunction and to physical disease (F6.0), depending upon the predominant clinical presentation and were referred for neurological evaluation. At the time of discharge, the diagnosis was changed to Mania in 9 out of $72(12.5 \%)$ cases followed by Schizophrenia in 5 out of 72 (6.94\%) cases and to Dissociative Disorder in 1 out of 72 cases (1.38\%). At 1 month follow up, the primary diagnosis of ATPD had changed in additional number of patients, including severe depressive episode with psychotic features in 1 out of 72 cases (1.38\%) and Alcohol Dependence syndrome with Alcohol induced psychotic disorder in 1 out of 72 cases (1.38\%).Similarly, at three months, the diagnosis of one more patient was revised to Cannabis induced psychotic disorder.

Table 2: Diagnosis at discharge and two follow-ups

\begin{tabular}{|l|l|l|l|}
\hline Diagnosis (N=72) & DischargeN(\%) & One monthN(\%) & Three months N(\%) \\
\hline ATPD & $54(75)$ & $52(72.2)$ & $48(66.7)$ \\
\hline Schizophrenia & $5(6.9)$ & $5(6.9)$ & $5(6.9)$ \\
\hline Mania & $9(12.5)$ & $9(12.5)$ & $9(12.5)$ \\
\hline Delirium or organic psychosis & $3(4.2)$ & $1(1.4)$ & $1(1.4)$ \\
\hline Dissociative disorder & $1(1.4)$ & $1(1.4)$ & $1(1.4)$ \\
\hline Depression with psychotic symptoms & - & $1(1.4)$ & $1(1.4)$ \\
\hline Alcohol induced psychotic disorder & - & $1(1.4)$ & $1(1.4)$ \\
\hline Cannabis induced psychotic disorder & - & - & $1(1.4)$ \\
\hline Lost to follow up/referred & - & $2(2.8)$ & $5(6.9)$ \\
\hline
\end{tabular}

\section{DISCUSSION}

Mean age of the sample population was 30.71 years which is similar to a study from $\operatorname{India}^{10}$ and comparable to the mean age of onset of 37.4 years in a study of Scottish Morbidity Record ${ }^{11}$ Though the international data suggests that the female are more prone to develop ATPD ${ }^{12,13}$, in our sample we found slight female preponderance like in the study by Mehta et $\mathrm{al}^{14}$. The majority of the study participants were from the rural areas (especially outside of Kathmandu valley) which may be because of the fact that this hospital is mostly visited by patients from the lower socioeconomic levels and mostly from the rural regions across the country. The mean duration of stay was 13.85 days and majority of them (68\%) staying less than 15 days is different to the findings from other studies. ${ }^{15,16}$ Though the duration of hospital stay is less in ATPD as compared to other psychosis, the duration from our study is lesser. ${ }^{17}$

The diagnostic stability of ATPD has been questioned since its inclusion in ICD-10. Castagnini et al. reported that about $50 \%$ of the cases with ATPD went on 
to develop 'schizophrenia and related disorders or affective disorders ${ }^{12}$. In developing countries, however, ATPD has a relatively high diagnostic stability (50-75\%). Sajith et al reported that $73 \%$ retained the original diagnosis of ATPD after 3 years. ${ }^{6}$ Similarly, good diagnostic stability was reported by Thangadurai et al. ${ }^{18}$ In a 12 -year follow-up study of acute psychosis from the Chandigarh DOSMeD cohort, 1 of the 17 cases developed schizophrenia, none developed affective disorders, and a majority retained the initial diagnosis of acute psychosis. ${ }^{19}$ In a short term study of diagnostic stability in South India as well, majority of the patients retained the original diagnosis, showing a good stability of $70.2 \%$ and $63.2 \%$ at 1 year and 2 year follow up respectively. ${ }^{10}$ In a previous Nepalese study also, the diagnosis of ATPD was retained in $80 \%$ of the cases at the end of 3 months follow up. ${ }^{20}$ In our study as well the original diagnosis of ATPD was retained in more than $65 \%$ of the cases at the end of 3 months which indicates a high diagnostic stability. Although the diagnosis of acute psychosis is stable in many cases, some patients may go on to develop schizophrenia or mood disorders (mainly bipolar disorders) over time. It is this group of patients who need to be studied further to identify factors predicting relapse into other disorders. Marneros et al. reported that $75 \%$ of cases with ATPD had a recurrent psychotic episode, 30\% developed affective disorders, and a relatively small number developed schizoaffective disorder or schizophrenia ${ }^{21}$ where as Jorgensen et al., reported of $15 \%$ of cases with ATPD developed schizophrenia and $28 \%$ had affective disorders ${ }^{7}$. In our study a higher number of patients had their diagnosis revised to Affective Disorders including Mania and Severe depression with psychotic features (13.88\%) when compared to Schizophrenia (6.94\%). This finding is in line with the published literatures. ${ }^{2}$

The major strength of our study is the reliability of the diagnosis as two consultant psychiatrist were involved in collecting data and making each diagnosis. Similarly, the drop out rate was very less and majority of patients turned up for the follow up. However, there are many limitations of the study. The duration of follow up in our study was just up to 3 months, which is a major limitation as the long term stability and outcome of the patients is still unknown. In addition, factors associated with revision of the initial diagnosis were not explored.

\section{Conclusion}

Despite limitations, our study highlights the diagnostic stability of acute and transient psychotic disorder in the Nepalese scenario, with two third of the patients retaining the initial diagnosis at 3 months. Future studies with longer duration of follow ups can shed more light on this topic in our scenario. Exploration of the factors associated with the evolution of ATPD into other psychiatric entities over time in our setting needs to be done.

\section{References}

1. Organization $\mathrm{WH}$. The ICD-10 classification of mental and behavioural disorders: clinical descriptions and diagnostic guidelines. World Health Organization, 1992.

2. Malhotra S. Acute and transient psychosis: A paradigmatic approach. Indian J Psychiatry 2007; 49: 233-243.

3. Organization $\mathrm{WH}$. Report of the international pilot study of schizophrenia.

4. Sartorius N, Jablensky A, Korten A, et al. Early manifestations and first-contact incidence of schizophrenia in different cultures. A preliminary report on the initial evaluation phase of the WHO Collaborative Study on determinants of outcome of severe mental disorders. Psychol Med 1986; 16: 909-928.

5. Cooper JE, Jablensky A, Sartorius N. WHO collaborative studies on acute psychoses using the SCAAPS schedule. Psychiatry World Perspect 1990; 1: 185-192.

6. Sajith SG, Chandrasekaran R, Sadanandan Unni $\mathrm{KE}$, et al. Acute polymorphic psychotic disorder: diagnostic stability over 3 years. Acta Psychiatr Scand 2002; 105: 104-109.

7. Jørgensen $P$, Bennedsen $B$, Christensen J, et al. Acute and transient psychotic disorder: a 1-year follow-up study. Acta Psychiatr Scand 1997; 96: 150-154.

8. Haahr U, Friis S, Larsen TK, et al. First-episode psychosis: diagnostic stability over one and two years. Psychopathology 2008; 41: 322-329.

9. Broome MR, Woolley JB, Tabraham P, et al. What causes the onset of psychosis? Schizophr Res 2005; 79: 23-34.

10. Narayanaswamy JC, Shanmugam VH, Raveendranathan $D$, et al. Short-Term Diagnostic Stability of Acute Psychosis: Data 
from a Tertiary Care Psychiatric Center in South India. Indian J Psychol Med 2012; 34: 176-178.

11. Queirazza F, Semple DM, Lawrie SM. Transition to schizophrenia in acute and transient psychotic disorders. Br J Psychiatry 2014; 204: 299-305.

12. Castagnini AC, Munk-Jørgensen P, Bertelsen A. Short-term course and outcome of acute and transient psychotic disorders: Differences from other types of psychosis with acute onset. Int J Soc Psychiatry 2016; 62: 51-56.

13. Susser $E$, Wanderling J. Epidemiology of nonaffective acute remitting psychosis vs schizophrenia. Sex and sociocultural setting. Arch Gen Psychiatry 1994; 51: 294-301.

14. Mehta S, Tyagi A, Swami MK, et al. Onset of acute and transient psychotic disorder in India: a study of socio-demographics and factors affecting its outcomes. East Asian Arch Psychiatry Off J Hong Kong Coll Psychiatr Dong Ya Jing Shen Ke Xue Zhi Xianggang Jing Shen Ke Yi Xue Yuan Qi Kan 2014; 24: 75-80.

15. Suda K, Hayashi N, Hiraga M. Predicting features of later development of schizophrenia among patients with acute and transient psychotic disorder. Psychiatry Clin Neurosci 2005; 59: 146-150.

16. Pillmann $F$, Haring $A$, Balzuweit $S$, et al. The concordance of ICD-10 acute and transient psychosis and DSM-IV brief psychotic disorder. Psychol Med 2002; 32: 525-533.

17. Jäger M D M, Hintermayr $M$, Bottlender R, et al. Course and outcome of first-admitted patients with acute and transient psychotic disorders (ICD-10:F23). Focus on relapses and social adjustment. Eur Arch Psychiatry Clin Neurosci 2003; 253: 209-215.

18. Thangadurai P, Gopalakrishnan R, Kurian S, et al. Diagnostic stability and status of acute and transient psychotic disorders. Br J Psychiatry J Ment Sci 2006; 188: 293.

19. Susser E, Varma VK, Mattoo SK, et al. Long-term course of acute brief psychosis in a developing country setting. Br J Psychiatry J Ment Sci 1998; 173: 226-230.

20. Ranjan S, Shakya R, Shyangwa PM. Diagnostic Stability of Acute and Transient Psychotic Disorders in Patients Attending Tertiary Care Hospital. J Univers Coll Med Sci 2015; 2: 7-10.

21. Marneros A, Pillmann F, Haring A, et al. Features of acute and transient psychotic disorders. Eur Arch Psychiatry Clin Neurosci 2003; 253: 167174. 